\title{
Non-Transferable Proxy Re-Encryption
}

\author{
Hui Guo, Zhenfeng Zhang and Jing Xu \\ Institute of Software, Chinese Academy of Sciences, Beijing, China. \\ \{guohui, zfzhang, xujing\}@tca.iscas.ac.cn
}

\begin{abstract}
Proxy re-encryption (PRE) allows a semi-trusted proxy to transform a ciphertext for Alice into a ciphertext of the same message for Bob. The traditional security notion of PRE focuses on preventing the proxy with the re-encryption key learning anything about the encrypted messages. However, such a basic security requirement is clearly not enough for many scenarios where the proxy can collude with Bob. A desirable security goal is therefore to prevent a malicious proxy colluding with Bob to re-delegate Alice's decryption right. In 2005, Ateniese, Fu, Green and Hohenberger first proposed this intriguing problem called non-transferability, in the sense that the only way for Bob to transfer Alice's decryption capability is to expose his own secret key. It captures the notion that Bob cannot collude with the proxy and transfer Alice's decryption right without compromising his own decryption capability. However, over the last decade, no solutions have achieved this property.

In this paper, we positively resolve this open problem. In particular, we give the first construction of nontransferable proxy re-encryption where the attacker is allowed to obtain one pair of keys consisting of Bob's secret key and the corresponding re-encryption key. Using indistinguishability obfuscation and $k$-unforgeable authentication as main tools, our scheme is provably secure in the standard model. The essential idea behind our approach is to allow Bob's secret key to be evoked in the process of decrypting Alice's ciphertext while hiding the fact that only Bob could decrypt it by the obfuscated program. In addition, we also show a negative result: a CPA secure proxy re-encryption scheme with "error-freeness" property cannot be non-transferable.
\end{abstract}

\section{Introduction}

In 1998, Blaze et al. [5] first proposed the primitive of proxy re-encryption (PRE) in order to solve the problem of delegating decryption rights. In a proxy re-encryption scheme, a semi-trusted proxy with reencryption keys can transform a ciphertext intended for Alice (delegator) into another ciphertext of the same plaintext intended for Bob (delegatee). The proxy cannot, however, learn anything about the underlying plaintext. According to the direction of transformation, PRE can be categorized into bidirectional PRE, in which the proxy can transform ciphertexts from Alice to Bob and vice versa, and unidirectional PRE, in which the proxy cannot transform ciphertexts in the opposite direction. There is also another method to classify PRE schemes, namely, a scheme is multi-hop, if the ciphertext can be transformed from Alice to Bob and then to Charlie and so on, otherwise it is single-hop. In this paper, we concentrate on single-hop unidirectional PRE.

In the last decade, proxy re-encryption has attracted many researchers' attention and has many intriguing applications, such as email forwarding [7], distributed files systems [1][2], digital rights management [23] and cloud data sharing [25]. In all these cases, the proxy is supposed to be semi-trusted and uncontrolled, that is, the traditional security notions prevent the adversary who has the re-encryption key from learning anything about the underlying messages. However, such a basic security requirement is clearly not enough for some applications. For instance, in cloud service scenarios, Alice may be a data broker and she sells sensitive data encrypted and stored in the cloud. Clearly, Alice has the data ownership and she does not hope anyone including the cloud access the data without her authorization. If Bob asks for her data, he has to pay first. Then Alice generates a re-encryption key for Bob and sends it to the cloud. With the re-encryption key, the cloud could transform the ciphertext for Bob. A desirable security goal is therefore to prevent a malicious cloud from colluding with Bob to further re-delegate Alice's decryption rights to another unpaid 
user Carol. Obviously, as soon as the cloud gets the re-encryption key, it could collude with Bob to decrypt Alice's ciphertext and forward the plaintext to Carol. However, this approach requires Bob to remain active and online. Again, Bob can always send his secret key to Carol, but in doing so, he assumes a security risk that is potentially injurious to himself.

In 2005, Ateniese et al. [1][2] first proposed this problem and introduced a notion of non-transferability. A proxy re-encryption scheme is said to be non-transferable if the proxy and a set of colluding delegatees cannot re-delegate decryption rights to other parties without compromising any malicious delegatee's decryption capability. This property can be seen as a tradeoff solution to protect Alice's "benefit" in delegating her decryption right: when a malicious delegatee Bob colludes with the proxy to transfer Alice's decryption capability to others, he must expose his own decryption capability as a pay. Informally speaking, non-transferability requires that the only way for Bob to transfer decryption capability of Alice is to expose his own secret key. More generally, if the proxy and Bob generate a useful decryption box $L_{a}$ in decrypting Alice's ciphertext, then $L_{a}$ is also useful in decrypting Bob's ciphertext.

Non-transferability of PRE scheme is an interesting problem in practice as illegal transfers of delegation may lead to financial loss and even worse, whereas the malicious user has very little risk of getting caught. Obviously, discouraging such behaviors seems much easier than preventing them, so one of the solutions is to trace the malicious proxy after its collusion with one or more delegatees. This means that the penalty can only be applied "after-the-fact", i.e., only after the unauthorized transfer has been finished. However, it is more desirable to have a better way to prevent collusion than to just discourage collusion. Non-transferability provides a proactive way of deterrence. In particular, if a malicious coalition transfers an unauthorized pirate decryption capability, any recipient will be able to obtain the decryption capability of the malicious delegatee. On the other hand, honest delegatees' decryption capabilities are guaranteed to remain hidden. How to construct a non-transferable PRE scheme is the main open problem left for PRE schemes.

Unfortunately, there are no solutions for this property since it was proposed. In fact, most known proposals for PRE allow the proxy colluding with Bob to directly generate new re-encryption keys from Alice to another user who has not been authorized by Alice, as pointed out in [13]. To achieve non-transferability, we need Alice's ciphertext has the property that only if a decryptor holds Bob's secret key (and the related re-encryption key), can it get the plaintext; otherwise, it will get nothing useful. In order to give a concrete construction, we resort to indistinguishability obfuscator $(i \mathcal{O})$ [3][12].

Indistinguishability obfuscation is a notion of general program obfuscation, proposed by Barak et al. [3], the target of which is to make computer programs unintelligible while not changing their functionality. Indistinguishability obfuscation requires obfuscations of any two (equal-size) programs that have the same functionality are computationally indistinguishable. Since Garg et al. [12] introduced the first candidate indistinguishability obfuscation, indistinguishability obfuscation becomes a very important tool to achieve unprecedented results. Unfortunately, their construction is built using some candidate graded encoding schemes [11] which are susceptible to the "zeroizing attacks" [11][10][16]. Recently, Lin [21] constructed a general-purpose indistinguishability obfuscation scheme for all polynomial-size circuits from only constant-degree graded encodings, which makes the zeroing attacks more difficult [10]. In this paper, we use generic indistinguishability obfuscation to resolve the problem of non-transferability in PRE.

\subsection{Our Contributions}

In this paper, we resolve the above open question and propose the first non-transferable PRE scheme. Our construction is conceptually very simple, and it uses indistinguishability obfuscation and $k$-unforgeable authentication [6] as main tools. Roughly, the ciphertexts are obfuscated programs and re-encryption keys are generated by the specific authentication scheme [6]. The proposed PRE scheme is CPA secure at the 2nd 
level ciphertext and the 1st level ciphertext. We prove that our scheme satisfies non-transferability, in the sense that the attacker is allowed to obtain one pair of keys consisting of a re-encryption key and the related delegatee's secret key, but it cannot transfer the delegator's decryption right without exposing the delegatee's secret key. It captures the most likely transference attack in reality: the proxy leaks the re-encryption keys carelessly and a malicious delegatee obtains the corresponding re-encryption key for himself.

Furthermore, we also demonstrate an interesting impossibility result in non-transferability. In particular we show that if a CPA secure PRE scheme is "error-free", then it cannot meet non-transferability. Informally, a PRE scheme is error-free if the re-encryption algorithm itself does not introduce any error to the reencrypted ciphertext. This notion is formalized by Zhang et al. [26] and used to achieve CCA secure PRE schemes. As far as we know almost all existing PRE schemes have error-freeness except the traceable PRE scheme in [19]. This result rules out the possibility of constructing error-free and non-transferable PRE schemes.

\subsection{Our Techniques}

When the proxy colludes with Bob, the proxy can use the relevant re-encryption key to transform Alice's ciphertext to another ciphertext intended for Bob, so it's clear that they are able to generate a pirate decryption box in decrypting any ciphertext of Alice. To obtain the property of non-transferability, the crucial difficulty is how to "extract" the decryption capability of Bob from the pirate decryption box. Generally speaking, when the process of re-encryption and decryption can easily be "obfuscated" into a program without any information about Bob's private key, it is impossible to achieve non-transferability. In this paper, we show both positive and negative results for the goal of constructing non-transferable PRE schemes.

The Negative Result. Zhang et al. formalized a strong correctness notion called "error-freeness" [26], which requires the re-encryption algorithm itself does not introduce any error to the re-encrypted ciphertext. That is, the correctness of re-encryption is preserved for all ciphertexts of Alice, even including malformed ciphertexts. With error-freeness, given any ciphertext for Alice and the corresponding re-encrypted ciphertext for Bob, Alice and Bob will always obtain the same message. Although this property is strongly desired in constructing CCA secure PRE schemes, it does conflict with the property of non-transferability.

To see this, consider the generic attack where the proxy colludes with Bob and derives a decryption box $L_{a}$ which is an obfuscation of the program: when given Alice's ciphertext, first re-encrypt it for Bob then decrypt the re-encrypted ciphertext using Bob's secret key. Due to error-freeness, $L_{a}$ is indistinguishable from the following obfuscated program: when given Alice's ciphertext, decrypt it using Alice's secret key. In the second obfuscated program, there is no need of Bob's secret key. Thus, intuitively we think that the decryption box $L_{a}$ hides all the private information of Bob and cannot be used to decrypt Bob's ciphertext. Furthermore, we also prove that it is impossible for a error-free PRE scheme to meet the non-transferability.

The Positive Result. To avoid the above impossibility result, our main goal is to give the first construction of non-transferable PRE.

In most previous works, although the colluding proxy and Bob cannot recover Alice's secret key, they can derive equivalent sub-keys that suffice to generate new re-encryption keys for unauthorized users, which means that these unauthorized users can decrypt all transformable ciphertexts intended for Alice. Obviously, such constructions cannot achieve non-transferability.

Motivated by this observation and the negative result, we propose a completely new approach to design non-transferable PRE schemes. In particular, a re-encryption key is an authentication which is generated by Alice to authenticate the identity of Bob, and both original ciphertexts and re-encrypted ciphertexts are the obfuscated programs which take as input the corresponding private key and output the plaintext. Since kunforgeable authentication scheme can only authenticate the designated user, even though the proxy colludes 
with Bob, they can not recover any useful information about Alice's private key and generate any new reencryption keys for other unauthorized users. Moreover, in order to avoid the mentioned negative result, we generate ciphertexts with "policies" such that for a malformed ciphertext and its re-encrypted ciphertext, Alice and Bob will get different plaintexts, which makes the transferability attack infeasible. Particularly, we use obfuscated programs to hide the plaintext and the ciphertext's "policies". A detailed description of our techniques follows.

For the construction of the ciphertexts, basically, our solution is to make Alice's ciphertext has the property that only if an adversary holds Bob's secret key $\mathrm{sk}_{b}$ and the related re-encryption key $\mathrm{rk}_{a \rightarrow b}$, can it get the plaintext $m$; otherwise, it will get nothing useful. Actually, it is somewhat like point-function obfuscation [4], however, it is not sufficient for realizing re-encryption functionality. Recall that in order to achieve non-transferability, Bob's decryption capability should be extracted from any illegal decryption box $L_{a}$ generated by the proxy and Bob. Particularly, our solution is to evoke $L_{a}$ to recover Bob's ciphertext and the main difficulties are how to embed Bob's ciphertext into a fake ciphertext of Alice for invoking $L_{a}$. Therefore, the ciphertext should have somewhat "self-contained" property and the resulting fake ciphertext should be indistinguishable from the normal one. To overcome these obstacles, we adopt the punctured programs technique [22] for applying indistinguishability obfuscation. In particular, the original ciphertext is two obfuscated programs: the first one, which is for Alice to decrypt, takes as input Alice's secret key and outputs the plaintext $m$; the second one, which is for re-encryption, verifies the validity of a re-encryption key and the corresponding delegatee's secret key and then outputs the plaintext $m$.

For the construction of the user's keys, basically, our solution is to make the re-encryption keys unforgeable. Otherwise, an adversary could transfer Alice's decryption right by forging a re-encryption key [13]. Furthermore, in order to use the security of indistinguishability obfuscation, the main difficulties are how to ensure the re-encryption keys to be information-theoretically unforgeable. Besides, the re-encryption keys should have a public verifiable algorithm for an encryptor to generate a ciphertext. To overcome the above difficulties, we adopt the specific authentication scheme in [6]. In particular, we regard an authentication, which is generated by Alice to authenticate Bob, as a re-encryption key.

Technique of Proof. For non-transferability, our primary challenge is to extract Bob's decryption capability from the illegal decryption box $L_{a}$ of Alice, which is generated by the collusion attack of proxy and Bob.

Our solution is to embed Bob's ciphertext in a fake ciphertext of Alice such that the fake ciphertext is polynomial indistinguishable from a normal ciphertext of the same encrypted message. With the fake ciphertext of Alice, we could run the illegal decryption box $L_{a}$ and get the underlying message. Unfortunately, though we use indistinguishability obfuscation to build the ciphertext, we could not directly obtain the indistinguishability of the fake ciphertext and the normal ciphertext. That is because, the programs being obfuscated in the fake ciphertext and the normal ciphertext have different functionalities. In particular, the normal ciphertext takes as input the secret key of any delegatee and the corresponding re-encryption key; while the fake ciphertext only takes as input Bob's secret key and the corresponding re-encryption key.

In order to show the indistinguishability of the fake ciphertext and the normal ciphertext by using the security of indistinguishability obfuscation, we build a hybrid experiment in which the normal ciphertext could not be evoked by pairs of keys $\left(\mathrm{rk}_{a \rightarrow b^{\prime}}, \mathrm{sk}_{b^{\prime}}\right)$ of any delegatee other than Bob. The key point to make this switch is that there does not exist such a key pair $\left(\mathrm{rk}_{a \rightarrow b^{\prime}}, \mathrm{sk}_{b^{\prime}}\right)$ leading to different outputs. In more detail, we introduce a fake key generation mode, which is also used in [6]. The scheme has an indistinguishable fake key generation mode, where there does not exist any secret keys of honest users (including Alice) or any re-encryption keys from Alice to corrupted users except the one from Alice to Bob (recall that Bob is a corrupted user). Under these restrictions, the fake ciphertext and the normal ciphertext 
would have the same inputs/outputs and they are indistinguishable by indistinguishability obfuscation's security. This also indicates that if a decryption box $L_{a}$ is useful in decrypting Alice's ciphertext, it must run with $\left(\mathrm{rk}_{a \rightarrow b}, \mathrm{sk}_{b}\right)$, thus Bob's decryption capability could be extracted from it.

\subsection{Related Works}

Since the notion of non-transferability was introduced in 2005, there have been many attempts to prevent the transferability of PRE. In 2008, Libert and Vergnaud [19] proposed traceable proxy re-encryption, where malicious proxies that reveal their re-encryption key to third parties can be identified by the delegator. Although it is one possible approach to the non-transferable PRE, it still cannot prevent colluding proxies and delegatees from re-delegating the decryption rights. It is more desirable to have a better way to prevent collusion, not just discourage collusion. In 2011, Wang et al. [24] proposed an identity-based proxy reencryption scheme to prevent collusion attacks. As the re-encryption key is generated using the master key of the PKG (a fully trusted private key generator), the proxy and the delegatees cannot further delegate the decryption right to others without the help of the PKG. However, this solution is undesirable since PKG in their scheme can decrypt both original and re-encrypted ciphertexts. Furthermore, PKG can transfer any re-encryption key for other users, that is, the transferable problem is still not solved. Later, Hayashi et al. [14] also introduced a relaxed notion of non-transferability and proposed two concrete constructions. Unfortunately, Isshiki et al. [17] pointed out that Hayashi et al.'s scheme is vulnerable to the forgeability attack of re-encryption keys. Moreover, the security assumption employed in their proofs can be solved efficiently. Later, Guo et al. [13] proposed an efficient PRE scheme with unforgeable re-encryption keys. However, they pointed out their security model could not capture all attacks of transference of decryption rights. Besides, there are several re-encryption constructions [8] [9] [15] achieving VBB obfuscation-based security. However, due to collusion attacks, they still cannot meet non-transferability.

\section{Preliminary}

In this section, we describe some primitives that will be used in our construction. Let $\lambda$ be the security parameter.

\subsection{Indistinguishability Obfuscation}

We recall the definition of indistinguishability obfuscator $(i \mathcal{O})$ [3][12]. It has been recently realized by Lin [21] from only constant-degree graded encodings under the assumption of constant-degree PRG and LWE, which makes the zeroing attacks more difficult as discussed in [10]. Note that our scheme is constructed from a generic $i \mathcal{O}$, so it is suitable for other possible $i \mathcal{O}$ instantiation.

Definition 1 (Indistinguishability Obfuscator). An indistinguishability obfuscator $i \mathcal{O}$ for a circuit class $\mathbb{C}_{\lambda}$ is a PPT uniform algorithm satisfying the following conditions:

- $i \mathcal{O}(\lambda, C)$ preserves the functionality of $C$. That is, for any $C \in \mathbb{C}_{\lambda}$, if we compute $C^{\prime}=i \mathcal{O}(\lambda, C)$, then $C^{\prime}(x)=C(x)$ for all inputs $x$.

- For any $\lambda$ and any two circuits $C_{0}, C_{1} \in \mathbb{C}_{\lambda}$ with the same functionality, the circuits $i \mathcal{O}\left(\lambda, C_{0}\right)$ and $i \mathcal{O}\left(\lambda, C_{1}\right)$ are indistinguishable. More precisely, for all pairs of PPT adversaries (Samp, D) there exists a negligible function $\alpha$ such that, if

$$
\operatorname{Pr}\left[\left(C_{0}, C_{1}, \tau\right) \leftarrow \operatorname{Samp}(\lambda): \forall x, C_{0}(x)=C_{1}(x)\right]>1-\alpha(\lambda)
$$


then

$$
\left|\operatorname{Pr}\left[\mathrm{D}\left(\tau, i \mathcal{O}\left(\lambda, C_{0}\right)\right)=1\right]-\operatorname{Pr}\left[\mathrm{D}\left(\tau, i \mathcal{O}\left(\lambda, C_{1}\right)\right)=1\right]\right|<\alpha(\lambda)
$$

In this paper, we will make use of such indistinguishability obfuscators for all polynomial-size circuits:

Definition 2 (Indistinguishability Obfuscator for P/poly). A uniform PPT machine $i \mathcal{O}$ is called an indistinguishability obfuscator for P/poly if the following holds: Let $\mathbb{C}_{\lambda}$ be the class of circuits of size at most $\lambda$. Then $i \mathcal{O}$ is an indistinguishability obfuscator for the class $\left\{\mathbb{C}_{\lambda}\right\}$.

\section{$2.2 k$-Unforgeable Authentication Scheme}

We recall the definition of $k$-unforgeable authentication recently realized in [6] using $k$-cover-free sets [18] and PRG. An authentication scheme over an identity space $\mathcal{I}$ consists of the following three algorithms:

- AuthGen $(\lambda)$ : The algorithm takes as input a security parameter $\lambda$, and outputs a secret authentication key ask and a public verification key avk.

- AuthProve(ask, id) : The algorithm takes as input a secret authentication key ask and a user's identity id, and outputs auth id $_{\text {. }}$

- Verify (avk, id, auth $\left.{ }_{\text {id }}\right)$ : The algorithm takes as input a public verification key avk, a user's identity id,

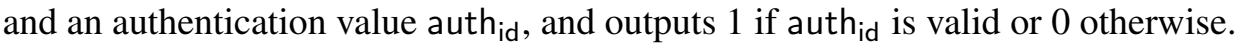

Correctness. For any identity id $\in \mathcal{I}$, the following condition holds:

$$
\operatorname{Pr}\left[(\text { avk, ask }) \leftarrow \operatorname{AuthGen}(\lambda) ; \text { auth }_{\text {id }} \leftarrow \text { AuthProve }(\text { ask, id }): \text { Verify }(\text { avk, id, auth } \text { id })=1\right]=1
$$

Definition 3 ( $k$-Unforgeability of Authentication Scheme). An authentication scheme is said to be $k$ unforgeable, if for any PPT adversary $\mathcal{A}$ and any set $\mathcal{T}$ of at most $k$ identities, the following condition holds:

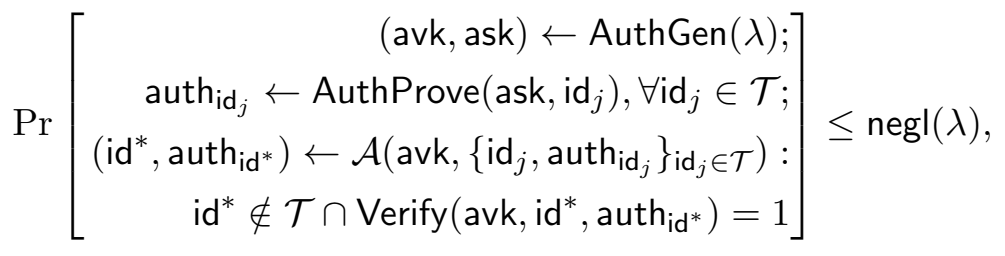

where $k$ is polynomially related to the security parameter.

Indistinguishable Fake Setup. The $k$-unforgeable authentication scheme constructed in [6] is accompanied with a PPT indistinguishable fake algorithm of setup. The algorithm FakeAuthGen $(\lambda, \mathcal{T})$ takes as input a security parameter $\lambda$ and a set $\mathcal{T}$ including $k$ identities, and outputs a key pair (ask, avk) such that it is only possible to authenticate the identities in $\mathcal{T}$ and there does not exist any authentication for users outside of $\mathcal{T}$. Moreover, the real setup and fake setup should be indistinguishable to the adversary. Formally, the security properties of algorithm FakeAuthGen are described as following:

- No authentication for id $\notin \mathcal{T}$ :

$$
\operatorname{Pr}\left[\begin{array}{r}
(\text { avk, ask }) \leftarrow \text { FakeAuthGen }(\lambda, \mathcal{T}): \\
\forall \text { id } \notin \mathcal{T}, \forall \text { auth }_{\text {id }} \in\{0,1\}^{*}, V_{\text {erify }}(\text { avk, id, auth } \\
\text { id })=0
\end{array}\right]>1-\operatorname{negl}(\lambda) .
$$


- Indistinguishability: For any PPT adversary $\mathcal{A}$, and any set $\mathcal{T}$ of at most $k$ identities, the following holds:

$$
\begin{aligned}
& \operatorname{Pr}\left[\begin{array}{r}
\text { (ask, avk }) \leftarrow \operatorname{AuthGen}(\lambda) ; \\
\text { auth }_{\mathrm{id}_{j}} \leftarrow \text { AuthProve }\left(\text { ask, } \text { id }_{j}\right), \forall \operatorname{id}_{j} \in \mathcal{T}: \\
\mathcal{A}\left(\text { avk, }\left\{\text { auth }_{\mathrm{id}_{j}}\right\}_{\left.\mathrm{id}_{j} \in \mathcal{T}\right)=1}\right.
\end{array}\right] \\
& \approx \operatorname{Pr}\left[\begin{array}{r}
(\text { ask, avk }) \leftarrow \text { FakeAuth } \operatorname{Gen}(\lambda, \mathcal{T}) ; \\
\text { auth }_{\mathrm{id}_{j}} \leftarrow \text { AuthProve }\left(\text { ask }_{1} \mathrm{id}_{j}\right), \forall \operatorname{id}_{j} \in \mathcal{T}: \\
\mathcal{A}\left(\text { avk, }\left\{\text { auth }_{\mathrm{id}_{j}}\right\}_{\left.\mathrm{id}_{j} \in \mathcal{T}\right)=1}\right.
\end{array}\right]
\end{aligned}
$$

\section{Proxy Re-Encryption}

In this section, we first recall the definitions of PRE. Then, we introduce the non-transferability definition against collusion attacks.

Let $\mathrm{M}$ denote the plaintext space. A single-hop unidirectional PRE scheme is a tuple of the following algorithms [1]:

- KeyGen $(\lambda)$ : The algorithm takes as input a security parameter $\lambda$, and outputs the user's public-secret key pair (pk, sk).

- ReKeyGen $\left(\mathrm{sk}_{i}, \mathrm{pk}_{j}\right)$ : The algorithm takes as input a secret key $\mathrm{sk}_{i}$ of user $i$ (i.e., delegator) and a public key $\mathrm{pk}_{j}$ of user $j$ (i.e., delegatee), and outputs a re-encryption key $\mathrm{rk}_{i \rightarrow j}$.

- Enc ${ }_{1}\left(\mathrm{pk}_{j}, m\right)$ : The algorithm takes as input a public key $\mathrm{pk}_{j}$ and a plaintext $m \in \mathrm{M}$, and outputs a first level ciphertext that cannot be re-encrypted for another user.

- Enc $2\left(\mathrm{pk}_{i}, m\right):$ The algorithm takes as input a public key $\mathrm{pk}_{i}$ and a plaintext $m \in \mathrm{M}$, and outputs a second level ciphertext that can be re-encrypted into a first level one (for a possibly different receiver) using the suitable re-encryption key.

- $\operatorname{ReEnc}\left(\mathrm{rk}_{i \rightarrow j}, C_{i}\right)$ : The algorithm takes as input a re-encryption key $\mathrm{rk}_{i \rightarrow j}$ and a second level ciphertext $C_{i}$, and outputs a first level ciphertext $C_{j}^{\prime}$ re-encrypted for user $j$.

- $\operatorname{Dec}_{1}\left(\mathrm{sk}_{j}, C_{j}^{\prime}\right):$ The algorithm takes as input a secret key sk ${ }_{j}$ and a first level ciphertext $C_{j}^{\prime}$, and outputs a plaintext $m \in \mathrm{M}$ or an error symbol $\perp$.

- $\operatorname{Dec}_{2}\left(\mathrm{sk}_{i}, C_{i}\right):$ The algorithm takes as input a secret key $\mathrm{sk}_{i}$ and a second level ciphertext $C_{i}$, and outputs a plaintext $m \in \mathrm{M}$ or an error symbol $\perp$.

Correctness. For any key pairs $\left(\mathrm{pk}_{i}, \mathrm{sk}_{i}\right),\left(\mathrm{pk}_{j}, \mathrm{sk}_{j}\right) \leftarrow \operatorname{KeyGen}(\lambda)$, any re-encryption key $\mathrm{rk}_{i \rightarrow j} \leftarrow$ ReKeyGen $\left(\mathrm{sk}_{i}, \mathrm{pk}_{j}\right)$ and any message $m \in \mathrm{M}$, the following conditions hold:

$$
\begin{gathered}
\operatorname{Dec}_{1}\left(\mathrm{sk}_{j}, \operatorname{Enc}_{1}\left(\mathrm{pk}_{j}, m\right)\right)=m ; \operatorname{Dec}_{2}\left(\mathrm{sk}_{i}, \operatorname{Enc}_{2}\left(\mathrm{pk}_{i}, m\right)\right)=m ; \\
\operatorname{Dec}_{1}\left(\mathrm{sk}_{j}, \operatorname{ReEnc}\left(\mathrm{rk}_{i \rightarrow j}, \operatorname{Enc}_{2}\left(\mathrm{pk}_{i}, m\right)\right)\right)=m .
\end{gathered}
$$




\subsection{CPA Security}

The security of second level ciphertexts for single-hop unidirectional PRE schemes [1] is defined as follows. Definition 4 (CPA Security of Second Level Ciphertext). A single-hop unidirectional PRE scheme is chosen plaintext secure at level 2 if

$$
\begin{array}{r}
\mid \operatorname{Pr}\left[\left\{\left(\mathrm{pk}_{j}, \mathrm{sk}_{j}\right) \leftarrow \operatorname{KeyGen}(\lambda)\right\}_{j \in \mathbb{C} \cup \mathbb{H}} ;\left(\mathrm{pk}_{i^{*}}, \mathrm{sk}_{i^{*}}\right) \leftarrow \operatorname{KeyGen}(\lambda)\right. \\
\left\{\mathrm{rk}_{i^{*} \rightarrow j} \leftarrow \operatorname{ReKeyGen}\left(\mathrm{sk}_{i^{*}}, \mathrm{pk}_{j}\right)\right\}_{j \in \mathbb{H}} ;\left\{\mathrm{rk}_{i \rightarrow j} \leftarrow \operatorname{ReKeyGen}\left(\mathrm{sk}_{i}, \mathrm{pk}_{j}\right)\right\}_{i \in \mathbb{H}, j \in \mathbb{C} \cup \mathbb{H} \cup\left\{i^{*}\right\}} ; \\
\left(m_{0}, m_{1}, s t\right) \leftarrow \mathcal{A}\left(\mathrm{pk}_{i^{*}},\left\{\mathrm{pk}_{j}, \mathrm{sk}_{j}\right\}_{j \in \mathbb{C}},\left\{\mathrm{pk}_{j}\right\}_{j \in \mathbb{H}},\left\{\mathrm{rk}_{i^{*} \rightarrow j}\right\}_{j \in \mathbb{H}},\left\{\mathrm{rk}_{i \rightarrow j}\right\}_{i \in \mathbb{H}, j \in \mathbb{C} \cup \mathbb{H} \cup\left\{i^{*}\right\}}\right) \\
\left.b \leftarrow\{0,1\} ; C^{*} \leftarrow \mathrm{Enc}_{2}\left(\mathrm{pk}_{i^{*}}, m_{b}\right) ; b^{\prime} \leftarrow \mathcal{A}\left(C^{*}, s t\right): b^{\prime}=b\right]-1 / 2 \mid \leq \operatorname{negl}(\lambda)
\end{array}
$$

for any PPT adversary $\mathcal{A}$, where the target user's key pair $\left(\mathrm{pk}_{j^{*}}, \mathrm{sk}_{j^{*}}\right)$ and the challenge ciphertext $C^{*}$ is generated by the challenger, st is the state information maintained by $\mathcal{A}$, and $\mathbb{C}$ and $\mathbb{H}$ denote the set of corrupted users and the set of honest users, respectively.

Remark 1. In the above security definition, we do not provide $\mathcal{A}$ with any re-encrypted ciphertext for the following two reasons: (1) $\mathcal{A}$ can generate re-encrypted ciphertexts from user $i^{*}$ to user $j(j \in \mathbb{H})$, and re-encrypted ciphertexts from user $i$ to user $j\left(i \in \mathbb{H}, j \in \mathbb{C} \cup \mathbb{H} \cup\left\{i^{*}\right\}\right.$ ), by using $\left\{\mathrm{rk}_{i^{*} \rightarrow j}\right\}_{j \in \mathbb{H}}$ and

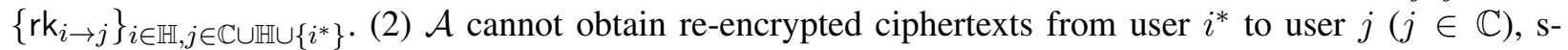
ince such ciphertexts implicitly provide the second level decryption capability of user $i^{*}$, which we do not consider in CPA security model.

Then we recall the security of first level ciphertexts for single-hop unidirectional PRE schemes. Since first level ciphertexts cannot be re-encrypted, the adversary $\mathcal{A}$ is granted access to all re-encryption keys in this definition.

Definition 5 (CPA Security of First Level Ciphertext). A single-hop unidirectional PRE scheme is chosen plaintext secure at level 1 if

$$
\begin{array}{r}
\mid \operatorname{Pr}\left[\left\{\left(\mathrm{pk}_{j}, \mathrm{sk}_{j}\right) \leftarrow \operatorname{KeyGen}(\lambda)\right\}_{j \in \mathbb{C} \cup \mathbb{H}} ;\left(\mathrm{pk}_{j^{*}}, \mathrm{sk}_{j^{*}}\right) \leftarrow \operatorname{KeyGen}(\lambda) ;\right. \\
\left\{\mathrm{rk}_{j \rightarrow j^{\prime}} \leftarrow \operatorname{ReKeyGen}\left(\mathrm{sk}_{j}, \mathrm{pk}_{j^{\prime}}\right)\right\}_{j, j^{\prime} \in \mathbb{C} \cup \mathbb{H} \cup\left\{j^{*}\right\}} ; \\
\left(m_{0}, m_{1}, s t\right) \leftarrow \mathcal{A}\left(\mathrm{pk}_{j^{*}},\left\{\mathrm{pk}_{j}, \mathrm{sk}_{j}\right\}_{j \in \mathbb{C}},\left\{\mathrm{pk}_{j}\right\}_{j \in \mathbb{H}},\left\{\mathrm{rk}_{j \rightarrow j^{\prime}}\right\}_{j, j^{\prime} \in \mathbb{C} \cup \mathbb{H} \cup\left\{j^{*}\right\}}\right) ; \\
\left.b \leftarrow\{0,1\} ; C^{*} \leftarrow \operatorname{Enc}_{1}\left(\mathrm{pk}_{j^{*}}, m_{b}\right) ; b^{\prime} \leftarrow \mathcal{A}\left(C^{*}, s t\right): b^{\prime}=b\right]-1 / 2 \mid \leq \operatorname{negl}(\lambda)
\end{array}
$$

for any PPT adversary $\mathcal{A}$, where the target user's key pair $\left(\mathrm{pk}_{j^{*}}, \mathrm{sk}_{j^{*}}\right)$ and the challenge ciphertext $C^{*}$ is generated by the challenger, st is the state information maintained by $\mathcal{A}$, and $\mathbb{C}$ and $\mathbb{H}$ denote the set of corrupted users and the set of honest users, respectively.

A PRE scheme is said to be CPA secure, if it is CPA secure at both level 1 and level 2.

\subsection{Non-Transferability}

Intuitively, non-transferability requires that the only way for a delegatee to transfer delegator's decryption capability is to expose his own second level decryption capability. To formalize this intuition, we first introduce a notion of $\epsilon$-useful second level decryption box, which is viewed as a PPT algorithm that takes as input a 2 nd level ciphertext and outputs a message or $\perp$. Such a decryption box does not need to be perfect, namely, we only require it to be able to decrypt with non-negligible success probability $\epsilon$.

Definition 6 ( $\epsilon$-Useful Second Level Decryption Box). For non-negligible probability value $\epsilon$, a PPT algorithm $L_{i, \epsilon}$ is an $\epsilon$-useful second level decryption box for user $i$, if $\operatorname{Pr}\left[m \leftarrow \mathrm{M}, C \leftarrow \operatorname{Enc}_{2}\left(\mathrm{pk}_{i}, m\right), m^{\prime} \leftarrow\right.$ $\left.L_{i, \epsilon}(C): m=m^{\prime}\right] \geq \epsilon$, where $\mathrm{M}$ is the plaintext space. 
Definition 7 (Non-Transferability). A single-hop unidirectional PRE scheme is non-transferable against collusion attacks if for any PPT adversary $\mathcal{A}$, there exists a PPT algorithm $I$ such that

$$
\begin{array}{r}
\operatorname{Pr}\left[\left\{\left(\mathrm{pk}_{j}, \mathrm{sk}_{j}\right) \leftarrow \operatorname{KeyGen}(\lambda)\right\}_{j \in \mathbb{C} \cup \mathbb{H}} ;\left(\mathrm{pk}_{i^{*}}, \mathrm{sk}_{i^{*}}\right) \leftarrow \operatorname{KeyGen}(\lambda) ;\right. \\
\left\{\mathrm{rk}_{i^{*} \rightarrow j} \leftarrow \operatorname{ReKeyGen}\left(\mathrm{sk}_{i^{*}}, \mathrm{pk}_{j}\right)\right\}_{j \in \mathbb{D}} ; \\
L_{i^{*}, \epsilon} \leftarrow \mathcal{A}\left(\mathrm{pk}_{i^{*}},\left\{\mathrm{pk}_{j}, \mathrm{sk}_{j}\right\}_{j \in \mathbb{C}},\left\{\mathrm{pk}_{j}\right\}_{j \in \mathbb{H}},\left\{\mathrm{rk}_{i^{*} \rightarrow j}\right\}_{j \in \mathbb{D}}\right): \\
\left.L_{j^{*}, \epsilon} \leftarrow I\left(L_{i^{*}, \epsilon}, \mathrm{pk}_{i^{*}},\left\{\mathrm{pk}_{j}\right\}_{j \in \mathbb{C} \cap \mathbb{D}}\right), j^{*} \in \mathbb{C} \cap \mathbb{D}\right]>1-\operatorname{negl}(\lambda)
\end{array}
$$

where $\mathcal{A}$ outputs an $\epsilon$-useful decryption box $L_{i^{*}, \epsilon}$ for user $i^{*}, I$ outputs an $\epsilon$-useful decryption box $L_{j^{*}, \epsilon}$ for user $j^{*}, \mathbb{C}$ denotes the set of corrupted users, $\mathbb{H}$ denotes the set of uncorrupted users, and $\mathbb{D} \subset \mathbb{C} \cup \mathbb{H}$ denotes the set of delegatees whose corresponding re-encryption keys are corrupted.

If $|\mathbb{C} \cap \mathbb{D}|=1$, the scheme is said to be non-transferable against one-pair-of-keys collusion attacks.

Remark 2. Non-transferability is considered as an advanced security requirement. In other words, it is meaningless for a PRE scheme to be non-transferable, unless the PRE scheme is CPA secure.

Remark 3. In our proposed scheme, the adversary $\mathcal{A}$ is allowed to obtain one pair of keys $\left(\mathrm{rk}_{i^{*} \rightarrow j}, \mathrm{sk}_{j}\right)$ in collusion attacks, e.g. $|\mathbb{C} \cap \mathbb{D}|=1$. Nevertheless, it captures the most common attack in reality: the proxy leaks the re-encryption keys carelessly, and a malicious delegatee obtains the corresponding re-encryption key for himself.

From another point of view, if $\mathcal{A}$ obtains more than one pair of keys, non-transferability might be hardly satisfied. Without loss of generality, $\mathcal{A}$ obtains $\left(\mathrm{rk}_{i^{*} \rightarrow j_{1}}, \mathrm{sk}_{j_{1}}\right)$ and $\left(\mathrm{rk}_{i^{*} \rightarrow j_{2}}, \mathrm{sk}_{j_{2}}\right)$, and there might be a decryption box $L_{i^{*}, \epsilon}^{\prime}$ with defending policy as follows. Taking a ciphertext as input, $L_{i^{*}, \epsilon}^{\prime}$ re-encrypts it to $C_{j_{1}}$ using $\mathrm{rk}_{i^{*} \rightarrow j_{1}}$ and decrypts $C_{j_{1}}$ using $\mathrm{sk}_{j_{1}}$; it repeats the re-encryption and the decryption by using $\left(\mathrm{rk}_{i^{*} \rightarrow j_{2}}, \mathrm{sk}_{j_{2}}\right)$, and it rejects if the two executions have different results. Intuitively, such $L_{i^{*}, \epsilon}^{\prime}$ might hardly be utilized in a black-box manner to decrypt either user $j_{1}$ 's or user $j_{2}$ 's ciphertexts.

Remark 4. In previous model [14], Bob and the proxy derive a re-encryption box $R$ which can re-encrypt Alice's ciphertext to another user Carol. In fact, a re-encryption box of [14] and a decryption box $L_{i^{*}, \epsilon}$ defined in this paper could be mutually transformed. If $L_{i^{*}, \epsilon}$ is leaked, we can use it to decrypt Alice's ciphertext and encrypt the message to Carol. If $R$ is leaked, we can use it to re-encrypt Alice's ciphertext and decrypt it with Carol's secret key (i.e. Carol use $R$ to extract Bob's decryption capability). The decryption box adopted in this paper is more simple and essential.

Remark 5. For convenience, we only consider the second level decryption capabilities of the malicious delegatees. Obviously, for delegatees, the second level ciphertext is more important while the first level ciphertext might be transformed ciphertext from other users.

\section{Construction}

In this section we present our construction of a non-transferable proxy re-encryption scheme.

Intuitively, to ensure the delegatee can decrypt the re-encrypted ciphertext using his own private key, the existing constructions require the delegator's private key "included" in the re-encryption key. Specifically, using the re-encryption key and the delegatee's private key, even though the delegator's private key itself cannot be disclosed, the adversary can obtain the partial information about the delegator's private key such as it's one-way transformation, using which the adversary can generate a new re-encryption key. Therefore, the scheme cannot be non-transferable. On the other hand, in almost all the existing constructions, the correctness of re-encryption is preserved for all ciphertexts of Alice. Thus, with the re-encryption key rk $\mathrm{r}_{a \rightarrow b}$ and the delegatee's private key $\mathrm{sk}_{b}$, the adversary can obfuscate the re-encryption algorithm $\operatorname{ReEnc}\left(\mathrm{rk}_{a \rightarrow b}, C_{a}\right)$ 
and decryption algorithm $\operatorname{Dec}_{1}\left(\mathrm{sk}_{b}, C_{b}^{\prime}\right)$ into a program $L_{a}$ which reveals no information about Bob's secret key $\mathrm{sk}_{b}$. More details can be found in Section 6. We believe that such attacks are the principle reasons that the existing constructions cannot provide non-transferability.

To overcome the above difficulties, we present a new methodology by applying the tools of indistinguishability obfuscation and k-unforgeable authentication. In our construction, a re-encryption key is an authentication which is generated by the delegator to authenticate the identity of a delegatee, and the ciphertexts (original and re-encrypted) are both the obfuscated programs which take as input the corresponding private key and output the plaintext. Since k-unforgeable authentication scheme can only authenticate the designated delegatee, even though the proxy colludes with a delegatee, they can not recover any useful information about the delegator's private key and generate any new re-encryption keys for other delegatees. Moreover, we use obfuscated program to generate ciphertexts with "policies" such that for a malformed ciphertext and its re-encrypted ciphertext, Alice and Bob will get different plaintext messages. The difference in the resulting messages makes the latter attack infeasible. In particular, the obfuscated program hides the plaintext and the ciphertext's "policies". This gives an intuition why our scheme achieves non-transferability.

Let Auth $=$ (AuthGen, AuthProve, Verify) be the $k$-unforgeable authentication scheme over an identity space $\mathcal{I}=\{0,1\}^{2 \lambda}$ of [6] ${ }^{1}, i \mathcal{O}$ be a program indistinguishability obfuscator, and PRG be a pseudo-random generator that maps $\{0,1\}^{\lambda}$ to $\{0,1\}^{2 \lambda}$. The message space $\mathcal{M}=\{0,1\}^{\lambda}$. The description of our single-hop unidirectional PRE scheme is given below.

- KeyGen $(\lambda)$ : Given the security parameter $\lambda$, the user $i$ randomly chooses $s_{i}$ and computes $t_{i}=$ $\operatorname{PRG}\left(s_{i}\right)$. It also generates $\left(\operatorname{avk}_{i}, \operatorname{ask}_{i}\right) \leftarrow \operatorname{AuthGen}(\lambda)$. Return the key pair $\left(\mathrm{pk}_{i}=\left(t_{i}, \mathrm{avk}_{i}\right), \mathrm{sk}_{i}=\right.$ $\left(s_{i}\right.$, ask $\left.\left._{i}\right)\right)$.

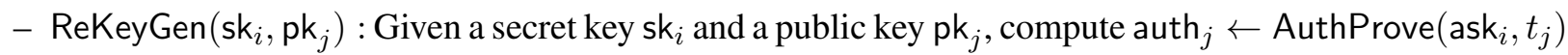
and set $\mathrm{rk}_{i \rightarrow j}=$ auth $_{j}$. Return the re-encryption key $\mathrm{rk}_{i \rightarrow j}$.

- Enc ${ }_{1}\left(\mathrm{pk}_{j}, m\right):$ Given a public key $\mathrm{pk}_{j}$, a message $m \in \mathcal{M}$, build the program Cipher-I in Figure 1. Return the ciphertext $C_{j}^{\prime}=i \mathcal{O}$ (Cipher-I). Note that $C_{j}^{\prime}$ is an obfuscated program, whose size is padded to be the maximum of itself and those of Figure 4 and Figure 5.

\section{Cipher-I}

Constants: $m, t_{j}$.

Input: $s_{j}$.

1. If $\operatorname{PRG}\left(s_{j}\right)=t_{j}$, output $m$ and exit.

2. Output “ $\perp$ ".

Fig. 1. The program Cipher-I

- $\mathrm{Enc}_{2}\left(\mathrm{pk}_{i}, m\right)$ : Given a public key $\mathrm{pk}_{i}$, a message $m \in \mathcal{M}$, build the programs Cipher-II-L and CipherII-R in Figure 2, and create $c_{l}=i \mathcal{O}$ (Cipher-II-L) and $c_{r}=i \mathcal{O}$ (Cipher-II-R). Return the ciphertext $C_{i}=\left(c_{l}, c_{r}\right)$. Note that $c_{l}$ and $c_{r}$ are two obfuscated programs, whose sizes are padded to the appropriate length.

\footnotetext{
${ }^{1}$ For any id $\in\{0,1\}^{2 \lambda}$, it can be interpreted as an element in $\mathbb{F}_{q}^{d+1}$, where $q=k \lambda, d=q-1 / k$, as required in [6]. In our scheme, $k$ is the bound of the number of corrupted re-encryption keys for a delegator.
} 


\section{Cipher-II-L}

Constants: $m, t_{i}$.

Input: $s_{i}$.

1. If $\operatorname{PRG}\left(s_{i}\right)=t_{i}$, output $m$ and exit.

2. Output “ $\perp$ ".

\section{Cipher-II-R}

Constants: $m$, avk $_{i}$.

Input: $\left(\mathrm{rk}_{i \rightarrow j}, s_{j}, t_{j}\right)$.

1. If Verify $\left(\mathrm{avk}_{i}, t_{j}, \mathrm{rk}_{i \rightarrow j}\right)=1$ and $\operatorname{PRG}\left(s_{j}\right)=t_{j}$ output $m$ and exit.

2. Output “ $\perp$ ".

Fig. 2. The programs Cipher-II-L and Cipher-II-R

- $\operatorname{ReEnc}\left(\mathrm{rk}_{i \rightarrow j}, \mathrm{pk}_{j},\left(c_{l}, c_{r}\right)\right)$ : Given a re-encryption key $\mathrm{rk}_{i \rightarrow j}$, a public key $\mathrm{pk}_{j}$ and a ciphertext $C_{i}=$ $\left(c_{l}, c_{r}\right)$, build the program Re-Cipher in Figure 3. Return the ciphertext $C_{j}^{\prime}=i \mathcal{O}$ (Re-Cipher). Note that $C_{j}^{\prime}$ is an obfuscated program, whose size is padded to the appropriate length.

\section{Re-Cipher}

Constants: $\mathrm{rk}_{i \rightarrow j}, t_{j}, c_{r}$.

Input: $s_{j}$.

1. Evaluate $c_{r}$ with $\left(\mathrm{rk}_{i \rightarrow j}, s_{j}, t_{j}\right)$ as input.

Fig. 3. The program Re-Cipher

- $\operatorname{Dec}_{1}\left(\mathrm{sk}_{j}, C_{j}^{\prime}\right)$ : Given a secret key sk ${ }_{j}$ and a first level ciphertext $C_{j}^{\prime}$, run the program $C_{j}^{\prime}$ on sk ${ }_{j}$ to obtain the plaintext $m$ or $\perp$.

- $\operatorname{Dec}_{2}\left(\mathrm{sk}_{i}, C_{i}\right)$ : Given a secret key sk ${ }_{i}$ and a second level ciphertext $C_{i}=\left(c_{l}, c_{r}\right)$, run the program $c_{l}$ on $\mathrm{sk}_{i}$ to obtain the plaintext $m$ or $\perp$.

Correctness. A second level ciphertext $C_{i}$ under $\mathrm{pk}_{i}$ has a form $\left(c_{l}, c_{r}\right)$ where $c_{l}$ and $c_{r}$ are two obfuscated programs. Given a re-encryption key rk ${ }_{i \rightarrow j}, C_{i}$ can be transformed into a first level ciphertext $C_{j}^{\prime}$ under pk , $_{j}$ where $C_{j}^{\prime}$ is also an obfuscated program. Also, the decryption algorithm runs the obfuscated program on inputting a secret key for either level ciphertext, the correctness is obvious.

\section{Security}

In this section, we prove the CPA security and non-transferability of the proposed construction.

\subsection{CPA Security}

First, we consider the CPA security.

Theorem 1. If PRG is a secure pseudo-random generator, Auth a k-unforgeable authentication scheme, and $i \mathcal{O}$ a secure indistinguishability obfuscator, then our PRE scheme is CPA secure at the first and second level ciphertext.

The theorem is obtained by combining the following Lemma 1 and Lemma 2. 
Lemma 1. If PRG is a secure pseudo-random generator and $i \mathcal{O}$ a secure indistinguishability obfuscator, then our PRE scheme is CPA secure at the first level ciphertext.

Proof. We describe a proof as a sequence of hybrid experiments where the first hybrid experiment corresponds to the original CPA security experiment of the 1st level ciphertext. We prove that the attacker $\mathcal{A}$ 's advantage must be negligibly close between each successive hybrid experiment and that the attacker has negligible advantage in the final experiment. Table 1 roughly describes the hybrid experiments.

- $\mathbf{H y b}_{0}$ The first hybrid is the real experiment.

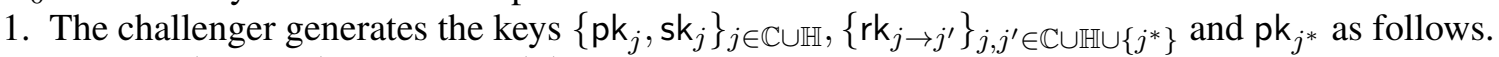

(a) Pick $\left(\mathrm{pk}_{j}, \mathrm{sk}_{j}\right) \leftarrow \operatorname{KeyGen}(\lambda), \forall j \in \mathbb{C} \cup \mathbb{H}$.

(b) Pick $s_{j^{*}}$ at random and compute $t_{j^{*}}=\operatorname{PRG}\left(s_{j^{*}}\right)$. Pick $\left(\mathrm{avk}_{j^{*}}, \mathrm{ask}_{j^{*}}\right) \leftarrow \operatorname{AuthGen}(\lambda)$. Set $\mathrm{pk}_{j^{*}}=\left(t_{j^{*}}, \mathrm{avk}_{j^{*}}\right)$.

(c) For all $j, j^{\prime} \in \mathbb{C} \cup \mathbb{H} \cup\left\{j^{*}\right\}$, generate $\mathrm{rk}_{j \rightarrow j^{\prime}} \leftarrow$ AuthProve $\left(\right.$ ask $\left._{j}, t_{j^{\prime}}\right)$.

It returns $\left\{\mathrm{pk}_{j}, \mathrm{sk}_{j}\right\}_{j \in \mathbb{C}},\left\{\mathrm{pk}_{j}\right\}_{j \in \mathbb{H}},\left\{\mathrm{rk}_{j \rightarrow j^{\prime}}\right\}_{j, j^{\prime} \in \mathbb{C} \cup \mathbb{H} \cup\left\{j^{*}\right\}}$ and $\mathrm{pk}_{j^{*}}$.

2. The attacker obtains $\left\{\mathrm{pk}_{j}, \mathrm{sk}_{j}\right\}_{j \in \mathbb{C}},\left\{\mathrm{pk}_{j}\right\}_{j \in \mathbb{H}},\left\{\mathrm{rk}_{j \rightarrow j^{\prime}}\right\}_{j, j^{\prime} \in \mathbb{C} \cup \mathbb{H} \cup\left\{j^{*}\right\}}$ and $\mathrm{pk}_{j^{*}}$. It chooses $m_{0}, m_{1} \in$ $\mathrm{M}$ and returns them to the challenger.

3. The challenger picks $b \in\{0,1\}$ at random, and generates the challenge ciphertext $C^{*}$ as an obfuscation of the program Target-Cipher-I in Figure 4.

4. $\mathcal{A}$ receives $C^{*}$ and outputs a guess $b^{\prime}$.

\section{Target-Cipher-I}

Constants: $m_{b}, t_{j^{*}}$.

Input: $s_{j^{*}}$.

1. If $\operatorname{PRG}\left(s_{j^{*}}\right)=t_{j^{*}}$, output $m_{b}$ and exit.

2. Output “ $\perp$ ".

Fig. 4. The program Target-Cipher-I

- $\mathbf{H y b}_{1}$ This hybrid experiment is the same as $\mathrm{Hyb}_{0}$ except that $t_{j^{*}}$ is randomly chosen.

- This hybrid experiment is indistinguishable from the previous one by the pseudo-randomness property of PRG.

- $\mathbf{H y b}_{2}$ This hybrid experiment is the same as $\mathrm{Hyb}_{1}$ except that we let the challenge ciphertext $C^{*}$ be an obfuscation of the program Target-Cipher-I* in Figure 5.

- Since $t_{j^{*}}$ is chosen at random, with high probability it is true $t_{j^{*}}$ is not in the image of the PRG and the program Target-Cipher-I will always output “ $\perp$ ". Therefore, the replacement will not change the functionality of the program Target-Cipher-I. Thus, the indistinguishability of $\mathrm{Hyb}_{1}$ and $\mathrm{Hyb}_{2}$ follows from the $i \mathcal{O}$ security property.

\begin{tabular}{c|c|c}
\hline Experiments & Description & Reduction to \\
\hline $\mathrm{Hyb}_{0}$ & the real experiment & - \\
\hline $\mathrm{Hyb}_{1}$ & $t_{j^{*}}$ is randomly chosen & security of PRG \\
\hline $\mathrm{Hyb}_{2}$ & $\begin{array}{c}\text { the challenge ciphertext } C^{*} \text { is an obfuscation } \\
\text { of the program Target-Cipher-I* in Figure 5 }\end{array}$ & $i \mathcal{O}$ security \\
\hline
\end{tabular}

Table 1. Description of the experiments in the proof of Lemma 1 


\section{Target-Cipher-I*}

Constants: $t_{j^{*}}$.

Input: $s_{j^{*}}$.

1. If $\operatorname{PRG}\left(s_{j^{*}}\right)=t_{j^{*}}$, output $\underline{0}$ and exit.

2. Output “ $\perp$ ".

Fig. 5. The program Target-Cipher-I*

In the last hybrid experiment, since the challenge ciphertext $C^{*}$ contains no information of $m_{b}$, the probability that the attacker will output a correct guess $b^{\prime}$ is negl $(\lambda)+1 / 2$.

Thus, the lemma follows.

Lemma 2. If PRG is a secure pseudo-random generator, Auth a k-unforgeable authentication scheme, and $i \mathcal{O}$ a secure indistinguishability obfuscator, then our PRE scheme is CPA secure at the second level ciphertext.

\begin{tabular}{c|c|c}
\hline Experiments & Description & Reduction to \\
\hline $\mathrm{Hyb}_{0}$ & the real experiment & - \\
\hline $\mathrm{Hyb}_{1}$ & use FakeAuthGen $\left(\lambda,\left\{t_{j}\right\}_{j \in \mathbb{H}}\right)$ for user $i^{*}$ & security of authentication \\
\hline $\mathrm{Hyb}_{2}$ & $t_{i}$ is chosen at random for all $i \in \mathbb{H} \cup\left\{i^{*}\right\}$ & security of PRG \\
\hline $\mathrm{Hyb}_{3}$ & $\begin{array}{c}\text { the challenge ciphertext } C^{*} \text { is the obfuscations of the programs } \\
\text { Target-Cipher-II-L* and Target-Cipher-II-R in Figure } 7\end{array}$ & $i \mathcal{O}$ security \\
\hline $\mathrm{Hyb}_{4}$ & $\begin{array}{c}\text { the challenge ciphertext } C^{*} \text { is the obfuscations of the programs } \\
\text { Target-Cipher-II-L* and Target-Cipher-II-R* in Figure 8 }\end{array}$ & $i \mathcal{O}$ security \\
\hline
\end{tabular}

Table 2. Description of the experiments in the proof of Lemma 2

Proof. We describe a proof as a sequence of hybrid experiments where the first hybrid experiment corresponds to the original CPA security experiment of the 2 nd level ciphertext. We prove that the attacker $\mathcal{A}$ 's advantage must be negligibly close between each successive hybrid experiment and that the attacker has negligible advantage in the final experiment. Table 2 roughly describes the hybrid experiments.

- $\mathbf{H y b}_{0}$ The first hybrid is the real experiment.

1. The challenger generates $\left\{\mathrm{pk}_{j}, \mathrm{sk}_{j}\right\}_{j \in \mathbb{C} \cup \mathbb{H}},\left\{\mathrm{rk}_{i^{*} \rightarrow j}\right\}_{j \in \mathbb{H}},\left\{\mathrm{rk}_{i \rightarrow j}\right\}_{i \in \mathbb{H}, j \in \mathbb{C} \cup \mathbb{H} \cup\left\{i^{*}\right\}}$ and $\mathrm{pk}_{i^{*}}$ as follows.

(a) For all $j \in \mathbb{C}$, pick $\left(\mathrm{pk}_{j}, \mathrm{sk}_{j}\right) \leftarrow \operatorname{KeyGen}(\lambda)$.

(b) For all $j \in \mathbb{H}$, pick $s_{j}$ at random and compute $t_{j}=\operatorname{PRG}\left(s_{j}\right)$. Pick $\left(\operatorname{avk}_{j}, \operatorname{ask}_{j}\right) \leftarrow \operatorname{AuthGen}(\lambda)$. Set $\mathrm{pk}_{j}=\left(t_{j}, \mathrm{avk}_{j}\right)$.

(c) For user $i^{*}$, pick $s_{i^{*}}$ at random and compute $t_{i^{*}}=\operatorname{PRG}\left(s_{i^{*}}\right)$. Pick $\left(\operatorname{avk}_{i^{*}}, \operatorname{ask}_{i^{*}}\right) \leftarrow \operatorname{AuthGen}(\lambda)$. Set $\mathrm{pk}_{i^{*}}=\left(t_{i^{*}}, \mathrm{avk}_{i^{*}}\right)$.

(d) For all $j \in \mathbb{H}$, generate $\mathrm{rk}_{i^{*} \rightarrow j} \leftarrow$ AuthProve $\left(\operatorname{ask}_{i^{*}}, t_{j}\right)$.

(e) For all $i \in \mathbb{H}, j \in \mathbb{C} \cup \mathbb{H} \cup\left\{i^{*}\right\}$, generate $\mathrm{rk}_{i \rightarrow j} \leftarrow$ AuthProve $\left(\right.$ ask $\left._{i}, t_{j}\right)$.

It returns $\left\{\mathrm{pk}_{j}, \mathrm{sk}_{j}\right\}_{j \in \mathbb{C}},\left\{\mathrm{pk}_{j}\right\}_{j \in \mathbb{H}},\left\{\mathrm{rk}_{i^{*} \rightarrow j}\right\}_{j \in \mathbb{H}},\left\{\mathrm{rk}_{i \rightarrow j}\right\}_{i \in \mathbb{H}, j \in \mathbb{C} \cup \mathbb{H} \cup\left\{i^{*}\right\}}$ and $\mathrm{pk}_{i^{*}}$.

2. The attacker receives $\left\{\mathrm{pk}_{j}, \mathrm{sk}_{j}\right\}_{j \in \mathbb{C}},\left\{\mathrm{pk}_{j}\right\}_{j \in \mathbb{H}},\left\{\mathrm{rk}_{i^{*} \rightarrow j}\right\}_{j \in \mathbb{H}},\left\{\mathrm{rk}_{i \rightarrow j}\right\}_{i \in \mathbb{H}, j \in \mathbb{C} \cup \mathbb{H} \cup\left\{i^{*}\right\}}$ and $\mathrm{pk}_{i^{*}}$. It chooses $m_{0}, m_{1} \in \mathrm{M}$ and returns them to the challenger. 
3. The challenger picks $b \in\{0,1\}$ at random and generate the challenge ciphertext $C^{*}=\left(c_{l}^{*}, c_{r}^{*}\right)$ as two obfuscations of the programs in Figure 6, separately.

4. $\mathcal{A}$ receives $C^{*}$ and outputs a guess $b^{\prime}$.

\section{Target-Cipher-II-L}

Constants: $m_{b}, t_{i^{*}}$.

Input: $s_{i^{*}}$.

1. If $\operatorname{PRG}\left(s_{i^{*}}\right)=t_{i^{*}}$, output $m_{b}$ and exit.

2. Output " $\perp$ ".

\section{Target-Cipher-II-R}

Constants: $m_{b}$, avk $_{i^{*}}$.

Input: $\left(\mathrm{rk}_{i^{*} \rightarrow j}, s_{j}, t_{j}\right)$.

1. If Verify $\left(\mathrm{avk}_{i^{*}}, t_{j}, \mathrm{rk}_{i^{*} \rightarrow j}\right)=1$ and $\operatorname{PRG}\left(s_{j}\right)=t_{j}$ output $m_{b}$ and exit.

2. Output " $\perp$ ".

Fig. 6. The programs Target-Cipher-II-L and Target-Cipher-II-R

- $\mathbf{H y b}_{1}$ This hybrid experiment is the same as $\mathrm{Hyb}_{0}$ except that we change the key generation algorithm for user $i^{*}$ to use FakeAuthGen $\left(\lambda,\left\{t_{j}\right\}_{j \in \mathbb{H}}\right)$ instead of AuthGen $(\lambda)$.

- This hybrid experiment is indistinguishable from the previous one by the security of the authentication scheme.

- $\mathbf{H y b}_{2}$ This hybrid experiment is the same as $\mathrm{Hyb}_{1}$ except that for all $i \in \mathbb{H} \cup\left\{i^{*}\right\}, t_{i}$ is randomly chosen. - The indistinguishability of $\mathrm{Hyb}_{2}$ from $\mathrm{Hyb}_{1}$ follows from the pseudo-randomness property of PRG.

- $\mathbf{H y b}_{3}$ This hybrid experiment is the same as $\mathrm{Hyb}_{2}$ except that we let the challenge ciphertext be the obfuscations of the programs in Figure 7.

- Since $t_{i^{*}}$ is chosen at random, with high probability it is true $t_{i^{*}}$ is not in the image of the PRG and the program Target-Cipher-II-L will always output " $\perp$ ". Therefore, the replacement will not change the functionality of the program Target-Cipher-II-L. Thus, the indistinguishability of $\mathrm{Hyb}_{3}$ and $\mathrm{Hyb}_{2}$ follows from the $i \mathcal{O}$ security property.

\section{Target-Cipher-II-L*}

Constants: $t_{i^{*}}$.

Input: $s_{i^{*}}$.

1. If $\operatorname{PRG}\left(s_{i^{*}}\right)=t_{i^{*}}$, output $\underline{0}$ and exit.

2. Output " $\perp$ ".

\section{Target-Cipher-II-R}

Constants: $m_{b}$, avk $_{i^{*}}$.

Input: $\left(\mathrm{rk}_{i^{*} \rightarrow j}, s_{j}, t_{j}\right)$.

1. If Verify $\left(\mathrm{avk}_{i^{*}}, t_{j}, \mathrm{rk}_{i^{*} \rightarrow j}\right)=1$ and $\operatorname{PRG}\left(s_{j}\right)=t_{j}$ output $m_{b}$ and exit.

2. Output “ $\perp$ ".

Fig. 7. The programs Target-Cipher-II-L* and Target-Cipher-II-R

- $\mathbf{H y b}_{4}$ This hybrid experiment is same as $\mathrm{Hyb}_{3}$ except that we let the challenge ciphertext be the obfuscations of the programs in Figure 8.

- Since $\left(\operatorname{avk}_{i^{*}}, \operatorname{ask}_{i^{*}}\right) \leftarrow$ FakeAuthGen $\left(\lambda,\left\{t_{j}\right\}_{j \in \mathbb{H}}\right)$, with high probability it is true that there is no authentication for user $j \notin \mathbb{H}$. Since for all $j \in \mathbb{H}, t_{j}$ is chosen at random, with high probability it is true $t_{j}$ is not in the image of the PRG. Therefore with high probability it is true the program Target-Cipher-II$\mathrm{R}$ will output " $\perp$ " and the replacement will not change the functionality of the program Target-CipherII-R. Thus, the indistinguishability of $\mathrm{Hyb}_{4}$ and $\mathrm{Hyb}_{3}$ follows from the $i \mathcal{O}$ security property.

In the last hybrid, since $C^{*}$ contains no information of $m_{b}$, the probability that the attacker will output a correct guess $b^{\prime}$ is $\operatorname{neg}(\lambda)+1 / 2$.

Thus, the lemma follows. 


\section{Target-Cipher-II-L*}

Constants: $t_{i^{*}}$.

Input: $s_{i *}$.

1. If PRG $\left(s_{i^{*}}\right)=t_{i^{*}}$, output 0 and exit.

2. Output “ $\perp$ ".

\section{Target-Cipher-II-R*}

Constants: avk $_{i^{*}}$.

Input: $\left(\mathrm{rk}_{i^{*} \rightarrow j}, s_{j}, t_{j}\right)$.

1. If $\operatorname{Verify}\left(\mathrm{avk}_{i^{*}}, t_{j}, \mathrm{rk}_{i^{*} \rightarrow j}\right)=1$ and $\operatorname{PRG}\left(s_{j}\right)=t_{j}$ output $\underline{0}$ and exit.

2. Output " $\perp$ ".

Fig. 8. The programs Target-Cipher-II-L* and Target-Cipher-II-R*

\subsection{Non-Transferability}

Theorem 2. If PRG is a secure pseudo-random generator, Auth a $k$-unforgeable authentication scheme, and $i \mathcal{O}$ a secure indistinguishability obfuscator, then our PRE scheme is non-transferable against one-pairof-keys collusion attacks.

\begin{tabular}{|c|c|c|c|c|}
\hline Keys & Ciphertext & Experiments & Description & Reduction to \\
\hline Normal & \multirow{3}{*}{ Fake } & $\mathrm{Hyb}_{0}$ & the real experiment & - \\
\hline \multirow{2}{*}{$\begin{array}{l}\text { Normal } \\
\rightarrow \text { Fake }\end{array}$} & & $\mathrm{Hyb}_{1}$ & use FakeAuthGen $\left(\lambda,\left\{t_{j}\right\}_{j \in \mathbb{D}}\right)$ for user $i^{*}$ & security of authentication \\
\hline & & $\mathrm{Hyb}_{2}$ & $t_{i}$ is chosen at random for all $i \in \mathbb{H} \cup\left\{i^{*}\right\}$ & security of PRG \\
\hline \multirow{3}{*}{ Fake } & \multirow{3}{*}{$\begin{array}{l}\text { Fake } \rightarrow \\
\text { Normal }\end{array}$} & $\mathrm{Hyb}_{3}$ & $\begin{array}{l}\text { the fake ciphertext } C_{f} \text { is the obfuscations of the programs } \\
\text { Fake-Cipher-II-L and Fake-Cipher-II-R* in Figure } 11\end{array}$ & $i \mathcal{O}$ security \\
\hline & & $\mathrm{Hyb}_{4}$ & $\begin{array}{l}\text { the fake ciphertext } C_{f} \text { is the obfuscations of the programs } \\
\text { Fake-Cipher-II-L and Fake-Cipher-II-R** } C_{f} \text { in Figure } 12\end{array}$ & $i \mathcal{O}$ security \\
\hline & & $\mathrm{Hyb}_{5}$ & $\begin{array}{l}\text { the fake ciphertext } C_{f} \text { is the obfuscations of the programs } \\
\text { Fake-Cipher-II-L* and Fake-Cipher-II-R** in Figure } 13\end{array}$ & $i \mathcal{O}$ security \\
\hline \multirow{2}{*}{$\begin{array}{l}\text { Fake } \rightarrow \\
\text { Normal }\end{array}$} & \multirow[t]{2}{*}{ Normal } & $\mathrm{Hyb}_{6}$ & $\begin{array}{c}t_{i}=\operatorname{PRG}\left(s_{i}\right) \text {, where } s_{i} \text { is randomly chosen, } \\
\text { for all } i \in \mathbb{H} \cup\left\{i^{*}\right\}\end{array}$ & security of PRG \\
\hline & & $\mathrm{Hyb}_{7}$ & use AuthGen $(\lambda)$ for user $i^{*}$ & security of authentication \\
\hline
\end{tabular}

Table 3. Description of the hybrid experiments in the proof of Theorem 2

Proof. The proposed construction allows one-pair-of-keys collusion attacks, that is $|\mathbb{C} \cap \mathbb{D}|=1$, where $\mathbb{C}$ denotes the set of corrupted users and $\mathbb{D}$ denotes the set of delegatees whose corresponding re-encryption keys are corrupted. Let $\left\{j^{*}\right\}=\mathbb{C} \cap \mathbb{D}$.

For any polynomial time algorithm $\mathcal{A}$ which outputs an $\epsilon$-useful decryption box $L_{i^{*}, \epsilon}$ for user $i^{*}$, we construct a PPT algorithm $L_{j^{*}, \epsilon}$ which uses $L_{i^{*}, \epsilon}$ as a subroutine to invert any given second level ciphertext $C^{*} \leftarrow \mathrm{Enc}_{2}\left(\mathrm{pk}_{j^{*}}, m\right)$ of user $j^{*}$ with probability $\epsilon$, where $m \leftarrow \mathrm{M}$.

To complete the proof, we describe the rest of the proof as a sequence of hybrid experiments and prove that $L_{j^{*}, \epsilon}$ 's advantage is negligibly close between each successive experiment. In the first hybrid experiment, the key generation is in the normal mode and the ciphertext for extracting $j^{*}$ 's decryption capability is a fake ciphertext. In $\mathrm{Hyb}_{1}$ and $\mathrm{Hyb}_{2}$, we change the key generation algorithm from the normal mode to the fake mode. In $\mathrm{Hyb}_{3}$ to $\mathrm{Hyb}_{5}$, the fake ciphertext is changed to a normal one. In $\mathrm{Hyb}_{6}$ and $\mathrm{Hyb}_{7}$, the key generation algorithms are back to the normal mode. In the final experiment, the decryption box $L_{j^{*}, \epsilon}$ would output the encrypted message $m$ with non-negligible probability $\epsilon$. Table 3 roughly describes the hybrid experiments.

- $\mathbf{H y b}_{0}$ The first hybrid experiment is the real experiment.

1. The challenger generates the keys $\left\{\mathrm{pk}_{j}, \mathrm{sk}_{j}\right\}_{j \in \mathbb{C}},\left\{\mathrm{pk}_{j}\right\}_{j \in \mathbb{H}},\left\{\mathrm{rk}_{i^{*} \rightarrow j}\right\}_{j \in \mathbb{D}}$ and $\mathrm{pk}_{i^{*}}$ as follows.

(a) For all $j \in \mathbb{C}$, pick $\left(\mathrm{pk}_{j}, \mathrm{sk}_{j}\right) \leftarrow \operatorname{KeyGen}(\lambda)$. 


\section{Target-Cipher-II-L}

Constants: $m, t_{j^{*}}$.

Input: $s_{j}$.

1. If $\operatorname{PRG}\left(s_{j^{*}}\right)=t_{j^{*}}$, output $m$ and exit.

2. Output " $\perp "$.

\section{Target-Cipher-II-R}

Constants: $m, \mathrm{avk}_{j^{*}}$.

Input: $\left(\mathrm{rk}_{j^{*} \rightarrow j^{\prime}}, s_{j^{\prime}}, t_{j^{\prime}}\right)$.

1. If $\operatorname{Verify}\left(\mathrm{avk}_{j^{*}}, t_{j^{\prime}}, \mathrm{rk}_{j^{*} \rightarrow j^{\prime}}\right)=1$ and $\operatorname{PRG}\left(s_{j^{\prime}}\right)=t_{j^{\prime}}$ output $m$ and exit.

2. Output “ $\perp "$.

Fig. 9. The programs Target-Cipher-II-L and Target-Cipher-II-R (of user $j^{*}$ )

\section{Fake-Cipher-II-L}

Constants: $0, t_{i^{*}}$.

Input: $s_{i^{*}}$.

1. If $\operatorname{PRG}\left(s_{i^{*}}\right)=t_{i^{*}}$, output 0 and exit.

2. Output “ $\perp ”$.

\section{Fake-Cipher-II-R}

Constants: $i \mathcal{O}$ (Target-Cipher-II-L), avk $i^{*}, t_{j^{*}}$.

Input: $\mathrm{rk}_{i^{*} \rightarrow j}, s_{j}, t_{j}$.

1. If $t_{j}=t_{j^{*}}$ and Verify $\left(\mathrm{avk}_{i^{*}}, t_{j}, \mathrm{rk}_{i^{*} \rightarrow j}\right)=1$, go to step 2; Else, output " $\perp$ " and exit.

2. Compute $i \mathcal{O}$ (Target-Cipher-II-L) with $s_{j}$ as input.

3. Output “ $\perp ”$.

Fig. 10. The programs Fake-Cipher-II-L and Fake-Cipher-II-R (of user $i^{*}$ )

(b) For all $j \in \mathbb{H}$, pick $s_{j}$ at random and compute $t_{j}=\operatorname{PRG}\left(s_{j}\right)$. Pick $\left(\operatorname{avk}_{j}, \operatorname{ask}_{j}\right) \leftarrow$ AuthGen $(\lambda)$. Set $\mathrm{pk}_{j}=\left(t_{j}, \mathrm{avk}_{j}\right)$.

(c) For user $i^{*}$, pick $s_{i^{*}}$ at random and compute $t_{i^{*}}=\operatorname{PRG}\left(s_{i^{*}}\right)$. Pick $\left(\operatorname{avk}_{i^{*}}, \operatorname{ask}_{i^{*}}\right) \leftarrow$ AuthGen $(\lambda)$. Set $\mathrm{pk}_{i^{*}}=\left(t_{i^{*}}, \mathrm{avk}_{i^{*}}\right)$.

(d) For all $j \in \mathbb{D}$, generate $\mathrm{rk}_{i^{*} \rightarrow j} \leftarrow$ AuthProve $\left(\operatorname{ask}_{i^{*}}, t_{j}\right)$.

It returns $\left\{\mathrm{pk}_{j}, \mathrm{sk}_{j}\right\}_{j \in \mathbb{C}},\left\{\mathrm{pk}_{j}\right\}_{j \in \mathbb{H}},\left\{\mathrm{rk}_{i^{*} \rightarrow j}\right\}_{j \in \mathbb{D}}$ and $\mathrm{pk}_{i^{*}}$.

2. $\mathcal{A}$ receives $\left\{\mathrm{pk}_{j}, \mathrm{sk}_{j}\right\}_{j \in \mathbb{C}},\left\{\mathrm{pk}_{j}\right\}_{j \in \mathbb{H}},\left\{\mathrm{rk}_{i^{*} \rightarrow j}\right\}_{j \in \mathbb{D}}$ and $\mathrm{pk}_{i^{*}}$. It outputs an $\epsilon$-useful decryption box $L_{i^{*}, \epsilon}$ of user $i^{*}$.

3. I generates a PPT algorithm $L_{j^{*}, \epsilon}$ which runs $L_{i^{*}, \epsilon}$ as a subroutine. It performs as follows.

(a) When given $C^{*}=(i \mathcal{O}$ (Target-Cipher-II-L), $i \mathcal{O}$ (Target-Cipher-II-R) $)$ as shown in Figure 9 where $m \leftarrow \mathrm{M}$, use $i \mathcal{O}$ (Target-Cipher-II-L) to generate the programs Fake-Cipher-II-L and Fake-Cipher-II-R in Figure 10.

(b) Obfuscate the programs and generate a fake ciphertext of user $i^{*}$

$$
C_{f}=(i \mathcal{O}(\text { Fake-Cipher-II-L }), i \mathcal{O}(\text { Fake-Cipher-II-R })) .
$$

(c) Run $L_{i^{*}, \epsilon}$ with $C_{f}$ as input and output what $L_{i^{*}, \epsilon}$ outputs.

- Hyb $\mathbf{b}_{1}$ This hybrid experiment is the same as $\mathrm{Hyb}_{0}$ except that we change the key generation algorithm for user $i^{*}$ to use FakeAuthGen $\left(\lambda,\left\{t_{j}\right\}_{j \in \mathbb{D}}\right)$ instead of AuthGen $(\lambda)$.

- This hybrid is indistinguishable from the previous one by the security of the authentication scheme.

- $\mathbf{H y b}_{2}$ This hybrid experiment is the same as $\mathrm{Hyb}_{1}$ except that $t_{i}$ is chosen at random for all $i \in \mathbb{H} \cup\left\{i^{*}\right\}$. - The indistinguishability of $\mathrm{Hyb}_{2}$ from $\mathrm{Hyb}_{1}$ follows from the pseudo-randomness property of PRG.

- $\mathbf{H y b}_{3}$ This hybrid experiment is the same as $\mathrm{Hyb}_{2}$ except that we let the fake ciphertext $C_{f}$ be the obfuscations of the programs Fake-Cipher-II-L and Fake-Cipher-II-R* in Figure 11. 
- In this hybrid experiment, we modify the fake ciphertext $C_{f}$ by replacing $i \mathcal{O}$ (Target-Cipher-II-L) in program Fake-Cipher-II-R with the corresponding unobfuscated program without changing its functionality. Thus, $\mathrm{Hyb}_{3}$ and $\mathrm{Hyb}_{2}$ are indistinguishable from the $i \mathcal{O}$ security.
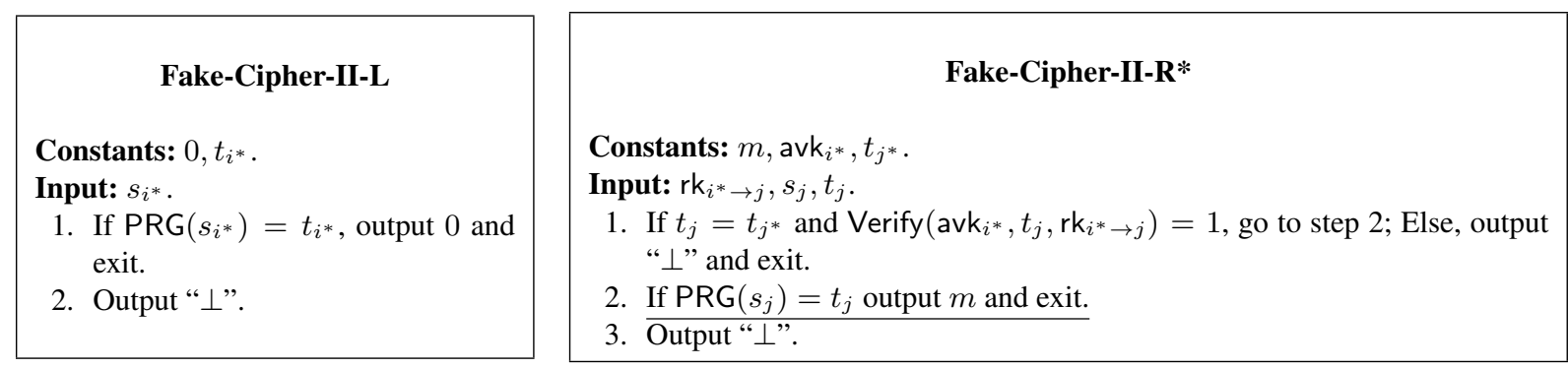

Fig. 11. The programs Fake-Cipher-II-L and Fake-Cipher-II-R* (of user $i^{*}$ )

- $\mathbf{H y b}_{4}$ This hybrid experiment is the same as $\mathrm{Hyb}_{3}$ except that we let the fake ciphertext $C_{f}$ be the obfuscation of the program Fake-Cipher-II-L and Fake-Cipher-II-R** in Figure 12.

- In this hybrid experiment, we remove the check $t_{j}=t_{j^{*}}$ in the program Fake-Cipher-II-R*. Since $\left(\operatorname{avk}_{i^{*}}, \operatorname{ask}_{i^{*}}\right) \leftarrow$ FakeAuthGen $\left(\lambda,\left\{t_{j}\right\}_{j \in \mathbb{D}}\right)$, with high probability it is true that there is no authentication for user $j \notin \mathbb{D}$. Since for all $j \in \mathbb{H}, t_{j}$ is chosen at random, with high probability it is true $t_{j}$ is not in the image of the PRG. Therefore with high probability it is true that only when $j \in \mathbb{C} \cap \mathbb{D}$ (i.e. $\left.t_{j}=t_{j^{*}}\right)$ could the tuple $\left(\mathrm{rk}_{i \rightarrow j}, s_{j}, t_{j}\right)$ pass the check $t_{j}=t_{j^{*}}$. Thus, the remove will not change the functionality of the program Fake-Cipher-II-R*. This hybrid is indistinguishable from the previous one by the $i \mathcal{O}$ security.

\section{Fake-Cipher-II-L}

Constants: $0, t_{i^{*}}$.

Input: $s_{i^{*}}$.

1. If PRG $\left(s_{i^{*}}\right)=t_{i^{*}}$, output 0 and exit.

2. Output " $\perp$ ".

\section{Fake-Cipher-II-R**}

Constants: $m$, avk $_{i^{*}}$.

Input: $\mathrm{rk}_{i^{*} \rightarrow j}, s_{j}, t_{j}$.

1. If Verify $\left(\mathrm{avk}_{i^{*}}, t_{j}, \mathrm{rk}_{i^{*} \rightarrow j}\right)=1$ and $\operatorname{PRG}\left(s_{j}\right)=t_{j}$ output $m$ and exit.

2. Output " $\perp$ ".

Fig. 12. The programs Fake-Cipher-II-L and Fake-Cipher-II-R** (of user $i^{*}$ )

- $\mathbf{H y b}_{5}$ This hybrid experiment is the same as $\mathrm{Hyb}_{4}$ except that we let the fake ciphertext $C_{f}$ be the obfuscation of the programs Fake-Cipher-II-L* and Fake-Cipher-II-R** in Figure 13.

- Since $t_{i^{*}}$ is chosen at random, with high probability it is true $t_{i^{*}}$ is not in the image of the PRG and the program Fake-Cipher-II-L will output “ $\perp$ ”. Therefore, the replacement will not change the functionality of the program Fake-Cipher-II-L. Thus, the indistinguishability of $\mathrm{Hyb}_{5}$ and $\mathrm{Hyb}_{4}$ follows from the $i \mathcal{O}$ security property.

- $\mathbf{H y b}_{6}$ This hybrid experiment is the same as $\mathrm{Hyb}_{5}$ except that for all $i \in \mathbb{H} \cup\left\{i^{*}\right\}$ we compute $t_{i}=$ $\operatorname{PRG}\left(s_{i}\right)$, where $s_{i}$ is randomly chosen.

- The indistinguishability of $\mathrm{Hyb}_{6}$ and $\mathrm{Hyb}_{5}$ follows from the pseudo-randomness property of PRG.

- $\mathbf{H y b}_{7}$ This hybrid experiment is same as $\mathrm{Hyb}_{6}$ except that we change the key generation algorithm for $i^{*}$ to use AuthGen $(\lambda)$. 


\section{Fake-Cipher-II-L*}

Constants: $m, t_{i^{*}}$.

Input: $s_{i}$.

1. If $\operatorname{PRG}\left(s_{i^{*}}\right)=t_{i^{*}}$, output $\underline{m}$ and exit.

2. Output “ $\perp$ ".

\section{Fake-Cipher-II-R**}

Constants: $m$, avk $_{i^{*}}$.

Input: $\mathrm{rk}_{i^{*} \rightarrow j}, s_{j}, t_{j}$.

1. If Verify $\left(\mathrm{avk}_{i^{*}}, t_{j}, \mathrm{rk}_{i^{*} \rightarrow j}\right)=1$ and PRG $\left(s_{j}\right)=t_{j}$ output $m$ and exit.

2. Output “ $\perp$ ".

Fig. 13. The programs Fake-Cipher-II-L* and Fake-Cipher-II-R** (of user $i^{*}$ )

- This hybrid is indistinguishable from the previous one by the security of the authentication scheme.

In the last hybrid, $C_{f}$ is a normal ciphertext that encrypts $m$ for user $i^{*}$. Since $L_{i^{*}, \epsilon}$ is an $\epsilon$-useful decryption box for user $i^{*}$, the probability that $L_{j^{*}, \epsilon}$ would output $m^{\prime}$ such that $m^{\prime}=m$ is $\epsilon$. Therefore, in the real experiment $L_{j^{*}, \epsilon}$ would be an $\epsilon$-useful second level decryption box with overwhelming probability.

Thus, the theorem follows.

\section{Impossibility Result for Non-Transferability}

In this section, we show that a PRE scheme which is error-free cannot achieve non-transferability.

The notion of error-freeness is a strong correctness requirement introduced by Zhang et al. [26] to achieve CCA security for PRE schemes. Many existing schemes such as [2][7][20] are actually error-free. It is also worth noting that error-freeness requires the correctness of re-encryption is preserved for all ciphertexts of Alice, even including malformed ciphertexts, while the correctness of a PRE scheme only requires the correctness of re-encryption is preserved for all valid ciphertexts of Alice.

Definition 8 (Error-Freeness). A PRE scheme $\Pi$ is said to be error-free, if for any second level ciphertext $C_{i}$ under $\mathrm{pk}_{i}, m=\operatorname{Dec}_{2}\left(\mathrm{sk}_{i}, C_{i}\right)$, and any re-encryption key $\mathrm{rk}_{i \rightarrow j}$, we have the probability

$$
\operatorname{Pr}\left[\operatorname{Dec}_{1}\left(\mathrm{sk}_{j}, \operatorname{ReEnc}\left(\mathrm{rk}_{i \rightarrow j}, C_{i}\right)\right)=m\right]=1 \text {. }
$$

Theorem 3. Under the assumption of indistinguishability obfuscators, for any CPA secure PRE scheme, if it is error-free, then it cannot be non-transferable.

Proof. Assume $\Pi^{\prime}=\left(\right.$ KeyGen', ReKeyGen', Enc ${ }_{1}^{\prime}$, Enc' $\left.2, \operatorname{ReEnc}^{\prime}, \operatorname{Dec}_{1}^{\prime}, \operatorname{Dec}_{2}^{\prime}\right)$ is a CPA secure and errorfree PRE scheme. Suppose user $i^{*}$ delegates his decryption rights to user $j^{*}$, and an adversary $\mathcal{A}$ corrupts the proxy and user $j^{*}$. $\mathcal{A}$ 's goal is to construct an $\epsilon$-useful decryption box $L_{i^{*}, \epsilon}$ such that $L_{i^{*}, \epsilon}$ doesn't contain any useful information for decrypting any ciphertext of user $j^{*}$.

When $\mathcal{A}$ obtains $\mathrm{pk}_{i^{*}}, \mathrm{pk}_{j^{*}}, \mathrm{sk}_{j^{*}}, \mathrm{rk}_{i^{*} \rightarrow j^{*}}$, it generates $L_{i^{*}, \epsilon}$ as the obfuscation of the program Transfer in Figure 14. It is obvious that $L_{i^{*}, \epsilon}$ is useful in decrypting user $i^{*}$ 's ciphertexts. The rest is to see that $L_{i^{*}, \epsilon}$ brings no advantages for decrypting $j^{*}$ 's ciphertexts. Consider another program $L_{i^{*}, \epsilon}^{\prime}$ which is an obfuscation of the program Transfer* in Figure 15.

The output $m=\operatorname{Dec}_{1}\left(\mathrm{sk}_{j^{*}}, \operatorname{ReEnc}\left(\mathrm{rk}_{i^{*} \rightarrow j^{*}}, C_{i^{*}}\right)\right)$ in the program Transfer is replaced by $m=\operatorname{Dec}_{2}\left(\mathrm{sk}_{i^{*}}, C_{i^{*}}\right)$ in the program Transfer*. Since $\Pi^{\prime}$ is error-free, the replacement would not change the functionality of the program Transfer. Thus, $L_{i^{*}, \epsilon}$ is indistinguishable from $L_{i^{*}, \epsilon}^{\prime}$ by the $i \mathcal{O}$ security.

Since $L_{i^{*}, \epsilon}^{\prime}$ is generated without $s_{j^{*}}$ and $\Pi^{\prime}$ is CPA secure, the probability of using $L_{i^{*}, \epsilon}^{\prime}$ to generate $j^{*}$ 's decryption box $L_{j^{*}, \epsilon}$ is negligible, which completes our proof. 


\section{Transfer}

Constants: $\mathrm{sk}_{j^{*}}, \mathrm{rk}_{i^{*} \rightarrow j^{*}}$.

Input: $C_{i^{*}}$.

1. Output $m=\operatorname{Dec}_{1}\left(\mathrm{sk}_{j^{*}}, \operatorname{ReEnc}\left(\mathrm{rk}_{i^{*} \rightarrow j^{*}}, C_{i^{*}}\right)\right)$.

Fig. 14. The program Transfer

\section{Transfer*}

Constants: $\mathrm{sk}_{i^{*}}$.

Input: $C_{i^{*}}$.

1. Output $\underline{m=\operatorname{Dec}_{2}\left(\mathrm{sk}_{i^{*}}, C_{i^{*}}\right)}$.

Fig. 15. The program Transfer*

\section{Conclusion}

Non-transferability is an intriguing problem both in practice and theory. In this paper, we formalized the notion of non-transferability and proposed the first non-transferable PRE construction based on indistinguishability obfuscation and $k$-unforgeable authentication scheme, which allows to leak one pair of keys consisting of a re-encryption key and the related delegatee's secret key. Indeed, the realization of nontransferable PRE scheme answers the open problem proposed by Ateniese et al. in 2005. Also, we proved the impossibility of non-transferability for "error-free" PRE schemes. Our negative result rules out a large class of construction methods for achieving non-transferability. Since this is the first step in formal investigation of non-transferable PRE, it would be interesting to see other methodologies for concrete constructions, e.g., getting rid of $i \mathcal{O}$.

\section{References}

1. Giuseppe Ateniese, Kevin Fu, Matthew Green, and Susan Hohenberger. Improved proxy re-encryption schemes with applications to secure distributed storage. In NDSS, 2005.

2. Giuseppe Ateniese, Kevin Fu, Matthew Green, and Susan Hohenberger. Improved proxy re-encryption schemes with applications to secure distributed storage. ACM Transactions on Information and System Security (TISSEC), 9(1):1-30, 2006.

3. Boaz Barak, Oded Goldreich, Rusell Impagliazzo, Steven Rudich, Amit Sahai, Salil Vadhan, and Ke Yang. On the (Im)possibility of obfuscating programs. In Advances in cryptology-CRYPTO 2001, pages 1-18. Springer, 2001.

4. Mihir Bellare and Igors Stepanovs. Point-function obfuscation: a framework and generic constructions. In Theory of Cryptography, pages 565-594. Springer, 2016.

5. Matt Blaze, Gerrit Bleumer, and Martin Strauss. Divertible protocols and atomic proxy cryptography. In Advances in Cryptology-EUROCRYPT 1998, pages 127-144. Springer, 1998.

6. Dan Boneh, Divya Gupta, Ilya Mironov, and Amit Sahai. Hosting services on an untrusted cloud. In Advances in CryptologyEUROCRYPT 2015, pages 404-436. Springer, 2015.

7. Ran Canetti and Susan Hohenberger. Chosen-ciphertext secure proxy re-encryption. In Proceedings of the 14th ACM conference on Computer and communications security, pages 185-194. ACM, 2007.

8. Nishanth Chandran, Melissa Chase, Feng-Hao Liu, Ryo Nishimaki, and Keita Xagawa. Re-encryption, functional reencryption, and multi-hop re-encryption: A framework for achieving obfuscation-based security and instantiations from lattices. In Public Key Cryptography-PKC 2014, pages 95-112. Springer, 2014. 
9. Nishanth Chandran, Melissa Chase, and Vinod Vaikuntanathan. Functional re-encryption and collusion-resistant obfuscation. In Theory of Cryptography, pages 404-421. Springer, 2012.

10. Jean-Sébastien Coron, Craig Gentry, Shai Halevi, Tancrede Lepoint, Hemanta K Maji, Eric Miles, Mariana Raykova, Amit Sahai, and Mehdi Tibouchi. Zeroizing without low-level zeroes: New MMAP attacks and their limitations. In Advances in Cryptology-CRYPTO 2015, pages 247-266. Springer, 2015.

11. Sanjam Garg, Craig Gentry, and Shai Halevi. Candidate multilinear maps from ideal lattices. In Advances in CryptologyEurocrypt 2013, pages 1-17. Springer, 2013.

12. Sanjam Garg, Craig Gentry, Shai Halevi, Mariana Raykova, Amit Sahai, and Brent Waters. Candidate indistinguishability obfuscation and functional encryption for all circuits. In Foundations of Computer Science (FOCS), 2013 IEEE 54th Annual Symposium on, pages 40-49. IEEE, 2013.

13. Hui Guo, Zhenfeng Zhang, and Jiang Zhang. Proxy re-encryption with unforgeable re-encryption keys. In Cryptology and Network Security, pages 20-33. Springer, 2014.

14. Ryotaro Hayashi, Tatsuyuki Matsushita, Takuya Yoshida, Yoshihiro Fujii, and Koji Okada. Unforgeability of re-encryption keys against collusion attack in proxy re-encryption. In Advances in Information and Computer Security, pages 210-229. Springer, 2011.

15. Susan Hohenberger, Guy N Rothblum, Abhi Shelat, and Vinod Vaikuntanathan. Securely obfuscating re-encryption. In Theory of Cryptography, pages 233-252. Springer, 2007.

16. Yupu Hu and Huiwen Jia. Cryptanalysis of GGH map. In Advances in Cryptology-EUROCRYPT 2016, pages 537-565. Springer, 2016.

17. Toshiyuki Isshiki, Manh Ha Nguyen, and Keisuke Tanaka. Attacks to the proxy re-encryption schemes from IWSEC2011. In Advances in Information and Computer Security, pages 290-302. Springer, 2013.

18. Ravi Kumar, Sridhar Rajagopalan, and Amit Sahai. Coding constructions for blacklisting problems without computational assumptions. In Advances in Cryptology-CRYPTO 1999, pages 609-623. Springer, 1999.

19. Benoît Libert and Damien Vergnaud. Tracing malicious proxies in proxy re-encryption. In Pairing-Based CryptographyPairing 2008, pages 332-353. Springer, 2008.

20. Benoît Libert and Damien Vergnaud. Unidirectional chosen-ciphertext secure proxy re-encryption. In Public Key Cryptography-PKC 2008, pages 360-379. Springer, 2008.

21. Huijia Lin. Indistinguishability obfuscation from constant-degree graded encoding schemes. In Advances in CryptologyEUROCRYPT 2016, pages 28-57. Springer, 2016.

22. Amit Sahai and Brent Waters. How to use indistinguishability obfuscation: Deniable encryption, and more. In Proceedings of the 46th Annual ACM Symposium on Theory of Computing, pages 475-484. ACM, 2014.

23. Gelareh Taban, Alvaro A Cárdenas, and Virgil D Gligor. Towards a secure and interoperable DRM architecture. In Proceedings of the ACM workshop on Digital rights management, pages 69-78. ACM, 2006.

24. Lihua Wang, Licheng Wang, Masahiro Mambo, and Eiji Okamoto. New identity-based proxy re-encryption schemes to prevent collusion attacks. In Pairing 2010, pages 327-346. Springer, 2010.

25. Lei $\mathrm{Xu}$, Xiaoxin $\mathrm{Wu}$, and Xinwen Zhang. A certificateless proxy re-encryption scheme for secure data sharing with public cloud. In Proceedings of the 7th ACM Symposium on Information, Computer and Communications Security, pages 1-10. ACM, 2012.

26. Jiang Zhang, Zhenfeng Zhang, and Yu Chen. PRE: Stronger security notions and efficient construction with non-interactive opening. Theoretical Computer Science, 542(3):1-16, 2014. 